\title{
Sarcopenia is associated with the neutrophil/ lymphocyte and platelet/lymphocyte ratios in operable gastric cancer patients: a prospective study
}

This article was published in the following Dove Press journal: Cancer Management and Research

\author{
Ji $\operatorname{Lin}^{1, *}$ \\ Weiteng Zhang ${ }^{1, *}$ \\ Yunshi Huang' \\ Weisheng Chen' \\ Ruisen $\mathrm{Wu}^{\prime}$ \\ Xiaodong Chen' \\ Neng Lou ${ }^{2}$ \\ Pengfei Wang'
}

'Department of Gastrointestinal Surgery, The First Affiliated Hospital,

Wenzhou Medical University,

Wenzhou, Zhejiang, China;

${ }^{2}$ Department of General Surgery,

Zhejiang University Huzhou Hospital,

Huzhou, Zhejiang, China

*These authors contributed equally to this work

\begin{abstract}
Purpose: Sarcopenia is distinguished by decreased skeletal muscle plus low muscle strength and/or physical performance. This study was designed to demonstrate the relationship between sarcopenia and systemic inflammatory response (neutrophil/lymphocyte ratio [NLR], platelet/ lymphocyte ratio [PLR], and large platelet/lymphocyte ratio [LPLR]) prior to radical gastrectomy for gastric cancer.

Patients and methods: We conducted a prospective study of gastric cancer patients who underwent radical gastrectomy. The clinical utility of the NLR, PLR, and LPLR was evaluated by receiver operating characteristic curves. Sarcopenia components including skeletal muscle index, handgrip strength, and $6 \mathrm{~m}$ usual gait speed were measured. Logistic analysis was used to identify the independent indices associated with sarcopenia.

Results: A total of 670 patients were included, representing 504 men and 166 women. Of these, 104 patients $(15.5 \%)$ were diagnosed with sarcopenia and 567 (84.5\%) were non-sarcopenia. PLR has a diagnostic sensitivity of $91.3 \%$ for sarcopenia. In addition to the indicators of preoperative age, nutritional risk screening, body mass index, preoperative albumin, and diabetes, the NLR and PLR were independent predictors for sarcopenia $(P<0.05)$.

Conclusion: The present study showed that at-diagnosis sarcopenia was associated with inflammation in patients with operable gastric cancer. Due to the complex assessment of muscle condition, PLR may be used as a primary screening test for sarcopenia. How systemic inflammatory response influences changes in sarcopenia may provide new therapeutic perception toward improving outcomes.
\end{abstract}

Keywords: gastric cancer, sarcopenia, neutrophil/lymphocyte ratio, platelet/lymphocyte ratio

\section{Introduction}

Gastric cancer is the fifth most frequently diagnosed cancer all over the world with one million new cases occurring every year. ${ }^{1}$ However, despite progress in tumor diagnosis, surgery, and drug therapy, the prognosis of gastric cancer remains poor. ${ }^{2,3}$ Malnutrition is common in gastric cancer patients consequent to inadequate digestion and nutrient absorption. ${ }^{4}$ In comparison, sarcopenia, an age-related syndrome associated with functional disturbance and disability, is distinguished by reduced skeletal muscle and low muscle strength plus physical performance. ${ }^{5}$ This condition can result due to many causes, such as malnutrition, aging, inactivity, inflammatory disease, and cancer. ${ }^{6}$ This syndrome has come up as a measure of the physiological reserve for the body. Besides age, body mass index (BMI), albumin, and comorbidities, ${ }^{7-10}$ previous studies confirmed
Departmentof General Surgery, Zhejiang University Huzhou Hospital, 198 Hongqi

Road, Huzhou, Zhejiang, China

Pengfei Wang

Department of Gastrointestinal Surgery, The First Affiliated Hospital, Wenzhou Medical University, Fanhaixi Road, Shangcai Village, Wenzhou, Zhejiang, China

Tel +8605775 5579443

Email wpfhawk@।26.com 
that sarcopenia influenced total postoperative complications and overall survival of gastric cancer patients. ${ }^{11,12}$

From the beginning of production of cancer cells to the occurrence of cancer cachexia, inflammation plays crucial role in each step of cancer progression.${ }^{13-15}$ Currently, the platelet/lymphocyte ratio (PLR) ${ }^{16}$ and neutrophil/lymphocyte ratio (NLR) ${ }^{17}$ are gradually gaining more attention as indicators of systemic inflammatory response (SIR). Several studies have revealed that PLR and NLR could be identified as indicators predictive of advanced stage, lymphatic metastasis, treatment response, and prognosis in some types of carcinoma. ${ }^{18-21}$ A pilot study has suggested that the accuracy of large platelet/lymphocyte ratio (LPLR) was superior to that of PLR in patients with cancer.$^{22}$ We assumed that SIR may be relevant in identifying patients with sarcopenia.

To our knowledge, however, no relevant research has elucidated the association between SIR and sarcopenia for gastric carcinoma, especially for operable gastric cancer. Furthermore, the majority of studies regarding sarcopenia have been retrospective and all have used skeletal muscle mass as the single diagnostic parameter of sarcopenia, neglecting the importance of muscle strength and physical ability, which constitute indispensable parts of sarcopenia as well.$^{23}$ Therefore, this prospective study was designed to analyze whether SIR, including PLR, NLR, and LPLR, is associated with sarcopenia in gastric cancer patients who undergo radical gastrectomy.

\section{Patients and methods}

\section{Study population}

We identified consecutive patients of the First Affiliated Hospital of Wenzhou Medical University, Zhejiang, China, who were diagnosed with operable gastric cancer between February 1, 2015 and December 31, 2016. The inclusion criteria were: 1) need to undergo radical gastrectomy; 2) performed preoperative CT examinations less than 2 weeks prior to the operation; and 3) able to walk and grip within 7 days before the operation. Considering the stable blood parameters, the exclusion criteria were the following: 1) occurrence of another malignancy during the 3 years prior to surgery; 2) an emergency operation; 3) preoperative chemotherapy or radiotherapy; 4) severe bleeding or immune system disease; 5) severe inflammation, such as secondary peritonitis. Based on the abovementioned criteria, 73 patients were excluded. Finally, 670 patients were included in this study.

\section{Preoperative factors}

All the data about patients were collected prior to surgery: 1) personal information, including age, sex, BMI, ASA grade (according to the standard proposed by the American Society of Anesthesiologists), and surgery history; 2) tumor characteristics, including tumor location, histological type, and TNM stage of tumor; and 3) blood routine index, including neutrophil, platelet, lymphocyte, and large platelet count. The histopathological types were defined as "differentiated (well/moderate)" or "undifferentiated (poor)". We performed nutritional risk screening (NRS-2002) within 24 hours of admission. Total score of 3 or greater than 3 were regarded as nutritional risk. ${ }^{24}$ The treatment for gastric cancer was based on the Japanese Gastric Cancer Treatment Guidelines $2010 .{ }^{25}$ Surgery history was defined as surgery that has been performed in the past including abdominal surgery.

\section{Definition of sarcopenia}

Based on the European Working Group on Sarcopenia ${ }^{23}$ and the Asian Working Group for Sarcopenia, ${ }^{26}$ low skeletal muscle mass plus low muscle strength were considered as sarcopenia.

A cross-sectional CT image of the third lumbar vertebra (L3) was selected for valuing muscle mass. ${ }^{27}$ Skeletal muscles were separated from other tissues by a Hounsfield units threshold range from 30 to $150 .{ }^{28}$ The muscles of L3 region include psoas, external obliques, internal obliques, erector spinae, quadratus lumborum, transversus abdominis, and rectus abdominis. Tissue borderlines were manually drawn out. To decrease the bias, one investigator who was blinded for the patient was well-trained to identify and measure the muscle area by a professional imaging software (INFINITT PACS software version 3.0.11.3 BN17 32 bit; INFINITT Healthcare Co., Ltd, Seoul, Korea). was well-trained to identify and measure the muscle area. L3 muscle cross-sectional areas computed from each image were normalized for height $\left(\mathrm{m}^{2}\right)$ to acquire the $\mathrm{L} 3$ skeletal muscle index ( $\left.\mathrm{L} 3 \mathrm{SMI}, \mathrm{cm}^{2} / \mathrm{m}^{2}\right){ }^{29}$

Being indispensable indices, preoperative grip strength and 6 meter usual gait speed were also determined, which, respectively, reflect muscle strength and physical performance. ${ }^{12}$ Grip strength was calculated by electronic hand dynamometer (EH101; Camry, Guangdong Province, China). Patients were asked to squeeze the device with all strength by using the dominant hand. Six-meter usual gait speeds were calculated by asking patients to walk through $6 \mathrm{~m}$, clocking from the first foot to the last foot over the finish line. The two tests were performed within 7 days before the operation. The maximal value of three consecutive tests was recorded.

In consideration of the ethnic differences between Asians and Europeans, ${ }^{23,26}$ sarcopenia was diagnosed based on the following parameters: 1 ) muscle mass (L3 SMI $\leq 40.8 \mathrm{~cm}^{2} / \mathrm{m}^{2}$ for men and $34.9 \mathrm{~cm}^{2} / \mathrm{m}^{2}$ for women); ${ }^{12}$ 2) muscle strength 
(hand grip strength $<26 \mathrm{~kg}$ for men and $<18 \mathrm{~kg}$ for women); and 3 ) muscle performance ( $6 \mathrm{~m}$ usual gait speed $<0.8 \mathrm{~m} / \mathrm{s}$ ). ${ }^{26}$

\section{SIR evaluation}

Blood specimens were prospectively gathered within 7 days before the operation and translocated to sterile centrifuge tubes, which were carefully delivered to the clinical laboratory department. The neutrophil, lymphocyte, platelet, and large platelet counts were calculated by a hemocounter (XE2100; Sysmex Co., Kobe, Japan). The maximal Youden index values were selected as the cutoff points for NLR, PLR, and LPLR in the resultant receiver operating characteristic (ROC) curves. ${ }^{30}$ The patients were divided into two groups, NLR and PLR, according to the cutoff points.

\section{Validation data for PLR}

According to the same criteria as described in the study population section, 162 patients were selected in our prospective database from January 2017 to June 2017 as validation group. The data about preoperative factors, definition of sarcopenia, and SIR evaluation were collected as mentioned earlier.

\section{Statistical analysis}

We carried out the Kolmogorov-Smirnov test to confirm the normality of continuous parameters, for example, the neutrophil, platelet, and lymphocyte counts, along with the NLR and PLR. The Mann-Whitney $U$ test was used to compare the non-normal distributed variables between the sarcopenia and non-sarcopenia groups. The performance of SIR in diagnosing sarcopenia was examined using the area under the ROC (AUROC) curves. The AUROC was expressed as plots of the test sensitivity vs 1-specificity. The sensitivity and specificity were also assessed. The chi-squared test was used to analyze the relationship between clinicopathological characteristics and the variables as well as in validation cohort. A multivariate logistic regression analysis was used to calculate the OR and 95\% CI of the confirmed independent variables, based on the univariate analysis results. A value of $P<0.05$ was regarded as statistically significant. SPSS software version 22.0 (SPSS Inc., Chicago, IL, USA) was used to perform statistical analyses.

\section{Results \\ Patient characteristics}

Of the 670 patients selected, 504 were men and 166 were women. The median age was 65.0 years (interquartile range 58.0-73.0). Hypoalbuminemia was found in 172 (25.7\%) patients. One hundred and four patients (15.5\%) exhibited sarcopenia and 567 (84.5\%) showed non-sarcopenia. Table 1 reports the detailed demographics and clinical characteristics of the selected patients.

Among the features of preoperative SIR markers in patients with sarcopenia, NLR $(P<0.001)$, PLR $(P<0.001)$, and LPLR $(P<0.001)$ were significantly higher in patients with gastric cancer and sarcopenia than in those with non-sarcopenia. Lymphocyte count $(P<0.001)$ was significantly lower in patients with gastric cancer and sarcopenia than in those with non-sarcopenia (Table 1). White blood cell, neutrophil, and platelet counts were not relevant with sarcopenia. The ROC curves were used to further evaluate the variables that differed

Table I Systemic inflammatory response markers according to sarcopenia (IQR)

\begin{tabular}{|c|c|c|c|c|}
\hline SIR markers & Total & Non-sarcopenia group & Sarcopenia group & $P$-value \\
\hline WBC count & $\begin{array}{l}6.22 \\
(4.85-7.22)\end{array}$ & $\begin{array}{l}6.19 \\
(4.85-7.29)\end{array}$ & $\begin{array}{l}6.36 \\
(4.82-7.09)\end{array}$ & 0.869 \\
\hline Neutrophil count & $\begin{array}{l}3.91 \\
(2.73-4.70)\end{array}$ & $\begin{array}{l}3.82 \\
(2.73-4.67)\end{array}$ & $\begin{array}{l}4.38 \\
(2.74-5.04)\end{array}$ & 0.066 \\
\hline Platelet count & $\begin{array}{l}248.01 \\
(191-288)\end{array}$ & $\begin{array}{l}246.49 \\
(191-286)\end{array}$ & $\begin{array}{l}256.31 \\
(187.75-291.75)\end{array}$ & 0.449 \\
\hline Lymphocyte count & $\begin{array}{l}1.64 \\
(1.21-1.99)\end{array}$ & $\begin{array}{l}1.70 \\
(1.28-2.06)\end{array}$ & $\begin{array}{l}1.35 \\
(0.92-1.62)\end{array}$ & $<0.001^{*}$ \\
\hline PLR & $\begin{array}{l}170.11 \\
(111.38-200.87)\end{array}$ & $\begin{array}{l}162.09 \\
(106.62-191.01)\end{array}$ & $\begin{array}{l}213.76 \\
(131.73-278.58)\end{array}$ & $<0.001^{*}$ \\
\hline NLR & $\begin{array}{l}2.72 \\
(1.70-3.16)\end{array}$ & $\begin{array}{l}2.51 \\
(1.62-3.02)\end{array}$ & \begin{tabular}{|l}
3.86 \\
$(2.04-4.00)$
\end{tabular} & $<0.00 I^{*}$ \\
\hline LPLR & $\begin{array}{l}48.39 \\
(31.65-57.75)\end{array}$ & $\begin{array}{l}45.88 \\
(31.16-54.67)\end{array}$ & $\begin{array}{l}62.01 \\
(38.00-78.30)\end{array}$ & $<0.001^{*}$ \\
\hline
\end{tabular}

Notes: *Statistically significant $(P<0.05)$.

Abbreviations: LPLR, large platelet/lymphocyte ratio; NLR, neutrophil/lymphocyte ratio; PLR, platelet/lymphocyte ratio; WBC, white blood cell; SIR, systemic inflammatory response; IQR, interquartile range. 
significantly. Figure 1 shows that the AUCs of preoperative NLR (0.663, 95\% CI 0.603-0.723), PLR (0.655, 95\% CI $0.598-0.712)$, and LPLR $(0.649,95 \%$ CI $0.587-0.712)$ were wider than those of the lymphocyte count $(0.321,95 \% \mathrm{CI}$ $0.266-0.375)$.

\section{Clinicopathological characteristics associated with SIR}

Based on the ROC curve shown in Figure 1, the cutoff values of the NLR, PLR, and LPLR for sarcopenia were set as 2.67, 116.85, and 59.41, respectively. According to the cutoff value, NLR sensitivity and specificity for sarcopenia diagnosis were $62.0 \%$ and $70.1 \%$, whereas those for PLR were $91.3 \%$ and $31.8 \%$, respectively. Groups of "high NLR (>2.67)" and "low NLR ( $\leq 2.67)$ " or "high PLR (>116.85)" and "low PLR ( $\leq 116.85)$ " were dichotomized. The data of groups of LPLR were not shown below. The number of patients with high NLR and high PLR was 236 (35.2\%) and 480 (71.6\%), respectively. The clinicopathological characteristics associated with PLR and NLR were analyzed further. The number of sarcopenia cases was statistically higher in the high PLR and NLR groups (both $P<0.001$ ) (Table 2). Concerning the other clinicopathological characteristics, high NLR was significantly associated with old age ( $>65$ years, $P=0.001)$, low BMI $\left(<18 \mathrm{~kg} / \mathrm{m}^{2}, P=0.043\right)$, high NRS $(>1, P<0.001)$, hypoalbuminemia ( $<35 \mathrm{~g} / \mathrm{L}, P<0.001)$, hypohemoglobinemia $(<100 \mathrm{~g} / \mathrm{L}, P<0.001)$, poor pathological type (undifferentiated, $P=0.045)$, high node status $(P<0.001)$, and depth of invasion $(P<0.001)$. PLR was significantly increased with older age $(P=0.005)$, high NRS $(P<0.001)$, hypoalbuminemia and hypohemoglobinemia $(P<0.001)$, poor pathological

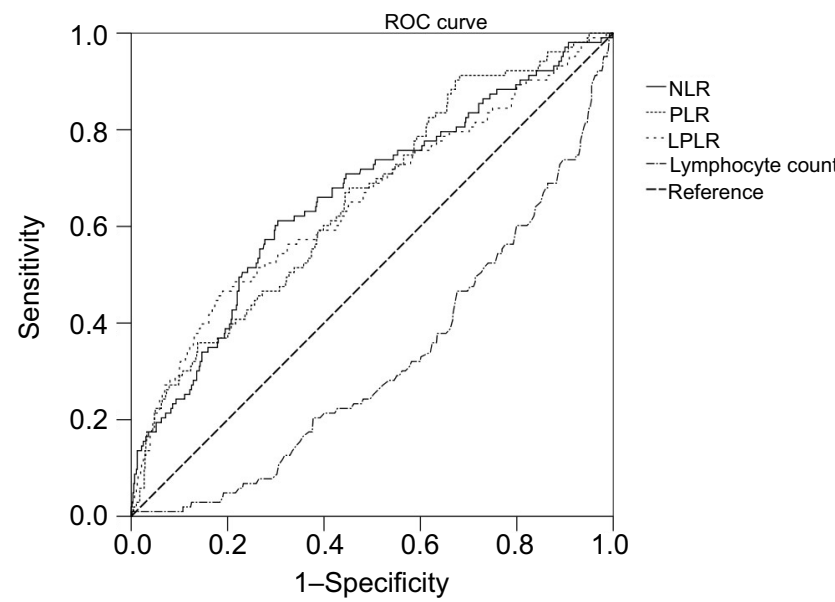

Figure I ROC curves for systemic inflammatory response makers in patients with gastric cancer according to sarcopenia.

Abbreviations: NLR, neutrophil/lymphocyte ratio; PLR, platelet/lymphocyte ratio; LPLR, large platelet/lymphocyte ratio; ROC, receiver operating characteristic. type $(P=0.05)$, depth of invasion $(P<0.001)$, and high node status $(P=0.008)$. No significant difference was observed with regard to the remainder of the clinicopathological characteristics.

\section{Univariate and multivariate analysis of clinicopathological characteristics}

Univariate analysis of clinicopathological characteristics indicated that age $(P<0.001)$, BMI $(P<0.001)$, ASA $(P=0.001)$, NRS $(P<0.001)$, surgery history $(P=0.036)$, histologic type $(P=0.042)$, hemoglobin $(P<0.001)$, diabetes $(P=0.006)$, albumin $(P<0.001)$, NLR $(P<0.001)$, PLR $(P<0.001)$, LPLR $(P<0.001)$, node status $(P=0.017)$, and $\mathrm{T}$ stage $(P<0.001)$ showed significant differences according to sarcopenia status (Table 3 ). Other variables such as sex and hypertension were found to have no significant association with sarcopenia.

Fourteen variables from univariate analysis $(P<0.05)$ were chosen for multivariate analysis as potential independent risk factors. Table 3 demonstrates that seven of the 14 variables differed significantly $(P<0.05)$. Finally, we confirmed that NLR (HR 2.065; 95\% CI 1.234-3.455; $P=0.006$ ), PLR (HR 2.194; 95\% CI 1.015-4.741; $P=0.046)$, age (HR 4.386; 95\% CI 2.297-8.376; $P<0.001$ ), NRS ([2 vs 1 ; HR 3.588; 95\% CI 1.171-10.996; $P=0.025$ ]; [3 vs 1 ; HR 7.246; 95\% CI 2.381-22.050; $P<0.001$ ]; [ $>3$ vs 1 ; HR 7.563; 95\% CI 2.46023.248; $P<0.001]$ ), albumin (HR 0.488; 95\% CI 0.292-0.815; $P=0.006$ ), BMI (HR 0.384; 95\% CI 0.162-0.90; $P=0.003$ ), and diabetes (HR 2.514; 95\% CI 1.269-4.984; $P=0.008$ ) were independent indicators predictive of sarcopenia, while ASA grade, surgical history, hemoglobin, histologic type, LPLR, tumor stage, and node status were not independently associated with sarcopenia.

\section{Validation cohort}

Considering sensitivity of PLR as an important outcome measure in this study, we externally validated PLR. Detailed patient characteristics are listed in Table 4. The cutoff value of PLR was defined as 116.85. The incidence in the group of sarcopenia was $16.7 \%$. PLR $(P=0.035)$ was significantly higher in patients with sarcopenia. PLR sensitivity of sarcopenia diagnosis was $88.9 \%$.

\section{Discussion}

This represents the first study to examine the relationship of SIR with sarcopenia in gastric cancer. In our cohort of 670 patients, we observed that greater NLR and PLR were associated with sarcopenia at diagnosis. The findings support 
Table 2 Clinical characteristics according to NLR and PLR (\%)

\begin{tabular}{|c|c|c|c|c|c|c|c|}
\hline Factors & $\begin{array}{l}\text { Total, } \\
670(\%)\end{array}$ & $\begin{array}{l}\text { Low NLR, } \\
434(\%)\end{array}$ & $\begin{array}{l}\text { High NLR, } \\
236(\%)\end{array}$ & $P$-value & $\begin{array}{l}\text { Low PLR, } \\
190(\%)\end{array}$ & $\begin{array}{l}\text { High PLR, } \\
480 \text { (\%) }\end{array}$ & $P$-value \\
\hline Age, years & & & & $0.00 I^{*}$ & & & $0.005^{*}$ \\
\hline$<65$ & $315(47.0)$ & $225(51.8)$ & $90(38.1)$ & & $106(55.8)$ & $209(43.5)$ & \\
\hline$\geq 65$ & $355(53.0)$ & $209(48.2)$ & $146(61.9)$ & & $84(44.2)$ & $271(56.5)$ & \\
\hline Gender & & & & 0.305 & & & 0.62 \\
\hline Male & $504(75.2)$ & $321(70.7)$ & $183(77.5)$ & & I 46 (76.8) & $358(74.6)$ & \\
\hline Female & $166(24.8)$ & I 33 (29.3) & $53(22.5)$ & & $44(23.2)$ & $122(25.4)$ & \\
\hline BMI, $\mathrm{kg} / \mathrm{m}^{2 \#}$ & & & & $0.043^{*}$ & & & 0.069 \\
\hline$<18$ & $40(6.0)$ & $20(4.6)$ & $20(8.5)$ & & $6(3.2)$ & $34(7.1)$ & \\
\hline$\geq 18$ & $628(94.0)$ & $413(95.4)$ & $215(91.5)$ & & $183(96.8)$ & 445 (92.9) & \\
\hline ASA & & & & 0.295 & & & 0.261 \\
\hline I & $86(12.8)$ & 59 (I3.6) & 27 (II.4) & & $2 I(I I . I)$ & 65 (I3.5) & \\
\hline 2 & 467 (69.7) & $308(71.0)$ & $159(67.4)$ & & $142(74.7)$ & $325(67.7)$ & \\
\hline 3 & $115(17.2)$ & $66(15.2)$ & $49(20.8)$ & & $26(13.7)$ & $89(18.6)$ & \\
\hline 4 & $2(0.3)$ & I (0.2) & $\mathrm{I}(0.4)$ & & I (0.5) & $\mathrm{I}(0.2)$ & \\
\hline NRS & & & & $<0.00 \mathrm{I}^{*}$ & & & $<0.001^{*}$ \\
\hline 1 & $212(31.6)$ & 157 (35.7) & $55(23.3)$ & & 81 (42.6) & I3I (27.3) & \\
\hline 2 & $186(27.8)$ & $131(29.8)$ & $55(23.3)$ & & $55(28.9)$ & $131(27.3)$ & \\
\hline 3 & $142(21.2)$ & 85 (19.3) & $57(24.2)$ & & $36(19.0)$ & $106(22.1)$ & \\
\hline$>3$ & $130(19.4)$ & 67 (I5.2) & $69(29.2)$ & & $18(9.5)$ & $112(23.3)$ & \\
\hline Diabetes & & & & 0.57 & & & 0.229 \\
\hline Yes & $76(11.3)$ & $47(10.2)$ & $29(12.3)$ & & $26(13.7)$ & $50(10.4)$ & \\
\hline No & 594 (88.7) & $387(89.8)$ & 207 (87.7) & & $164(86.3)$ & $430(89.6)$ & \\
\hline Hypertension & & & & 0.104 & & & 0.062 \\
\hline No & $499(74.5)$ & $332(76.5)$ & $167(70.8)$ & & 151 (79.5) & $348(72.5)$ & \\
\hline Yes & $171(25.5)$ & $102(23.5)$ & $69(29.3)$ & & $39(20.5)$ & $132(27.5)$ & \\
\hline Albumin, $g / L$ & & & & $<0.00 \mathrm{I}^{*}$ & & & $<0.001^{*}$ \\
\hline$<35$ & $172(25.7)$ & 79 (I8.2) & 93 (39.4) & & $21(11.7)$ & $15 \mid(31.5)$ & \\
\hline$\geq 35$ & $498(74.3)$ & $355(81.8)$ & $143(60.6)$ & & $169(89.3)$ & $329(68.5)$ & \\
\hline Hemoglobin, g/L & & & & $<0.00 \mathrm{I}^{*}$ & & & $<0.001^{*}$ \\
\hline$<100$ & $137(20.4)$ & $58(13.4)$ & $79(33.5)$ & & $9(4.7)$ & $128(26.7)$ & \\
\hline$\geq 100$ & $533(79.6)$ & $376(86.6)$ & $157(66.5)$ & & 181 (95.3) & $352(73.3)$ & \\
\hline Histologic type & & & & $0.045^{*}$ & & & $0.05^{*}$ \\
\hline Differentiated & $226(33.8)$ & $158(36.5)$ & $68(28.8)$ & & $75(39.5)$ & $151(31.5)$ & \\
\hline Undifferentiated & $443(66.2)$ & $275(63.5)$ & $168(7 \mid .2)$ & & $115(60.5)$ & $328(68.5)$ & \\
\hline Node status ${ }^{\#}$ & & & & $<0.00 \mathrm{I}^{*}$ & & & $0.008^{*}$ \\
\hline No & $302(45.6)$ & $219(50.8)$ & $83(35.9)$ & & $103(54.8)$ & $199(42.0)$ & \\
\hline NI & $117(17.7)$ & $66(15.3)$ & $5 I(22.1)$ & & $30(16.0)$ & $87(18.4)$ & \\
\hline N2 & $127(19.2)$ & $83(19.3)$ & 44 (19.1) & & $34(18.1)$ & $93(19.6)$ & \\
\hline N3 & $116(17.5)$ & $63(14.6)$ & $53(22.9)$ & & $21(11.1)$ & $95(2.0)$ & \\
\hline T stage st $^{\prime}$ & & & & $<0.00 I^{*}$ & & & $<0.001^{*}$ \\
\hline Tis/TI & $173(26.2)$ & $135(31.4)$ & $38(16.5)$ & & $82(43.2)$ & 91 (19.3) & \\
\hline $\mathrm{T} 2$ & $79(11.9)$ & $59(13.7)$ & $20(8.6)$ & & $23(12.1)$ & $56(11.9)$ & \\
\hline $\mathrm{T} 3$ & $142(21.5)$ & 84 (19.5) & $58(25.1)$ & & $27(14.2)$ & $115(24.4)$ & \\
\hline T4 & $267(40.4)$ & $152(35.4)$ & $115(49.8)$ & & $58(30.5)$ & $209(44.4)$ & \\
\hline Sarcopenia & & & & $<0.001^{*}$ & & & $<0.001^{*}$ \\
\hline No & $566(84.5)$ & 395 (91.0) & I7| (72.5) & & 179 (94.2) & $387(80.6)$ & \\
\hline Yes & $104(15.5)$ & $39(9.0)$ & $65(27.5)$ & & II (5.8) & $93(19.4)$ & \\
\hline $\begin{array}{l}\text { Muscle mass, } \mathrm{cm}^{2} / \mathrm{m}^{2} \\
\text { (IQR) }\end{array}$ & $\begin{array}{l}42.26 \\
(35.92-47.74)\end{array}$ & $\begin{array}{l}43.18 \\
(37.24-48.21)\end{array}$ & \begin{tabular}{|l|}
40.59 \\
$(34.46-46.21)$
\end{tabular} & & $\begin{array}{l}44.59 \\
(39.18-50.17)\end{array}$ & $\begin{array}{l}41.35 \\
(35.23-46.78)\end{array}$ & \\
\hline $\begin{array}{l}\text { Grip strength, kg } \\
\text { (IQR) }\end{array}$ & $\begin{array}{l}28.59 \\
(21.60-35.50)\end{array}$ & $\begin{array}{l}29.42 \\
(22.48-36.43)\end{array}$ & \begin{tabular}{|l|}
27.07 \\
$(20.47-34.30)$
\end{tabular} & & $\begin{array}{l}30.52 \\
(23.08-37.55)\end{array}$ & $\begin{array}{l}27.83 \\
(21.08-34.70)\end{array}$ & \\
\hline $\begin{array}{l}\text { Gait speed, m/s } \\
\text { (IQR) }\end{array}$ & $\begin{array}{l}1.01 \\
(0.87-1.13)\end{array}$ & $\begin{array}{l}1.02 \\
(0.89-1.14)\end{array}$ & $\begin{array}{l}0.98 \\
(0.81-1.12)\end{array}$ & & $\begin{array}{l}1.00 \\
(0.88-1.14)\end{array}$ & $\begin{array}{l}1.01 \\
(0.87-1.13)\end{array}$ & \\
\hline
\end{tabular}

Notes: The values given are number of patients unless indicated otherwise. *Statistically significant $(P<0.05)$; ${ }^{*}$ the data are partially lost for various reasons.

Abbreviations: ASA, American Society of Anesthesiologists; BMI, body mass index; IQR, interquartile range; NLR, neutrophil/lymphocyte ratio; NRS, nutritional risk screening; PLR, platelet/lymphocyte ratio. 
Table 3 Univariate analysis and multivariate analysis of the risk of sarcopenia

\begin{tabular}{|c|c|c|c|c|}
\hline \multirow[t]{2}{*}{ Factors } & \multirow[t]{2}{*}{$\begin{array}{l}\text { Non-sarcopenia } \\
\text { group, } 566(\%)\end{array}$} & \multirow[t]{2}{*}{$\begin{array}{l}\text { Sarcopenia } \\
\text { group, } 104 \text { (\%) }\end{array}$} & \multirow{2}{*}{$\begin{array}{l}\begin{array}{l}\text { Univariate } \\
\text { analysis }\end{array} \\
P \text {-value } \\
\end{array}$} & \multirow{2}{*}{$\begin{array}{l}\text { Multivariate } \\
\text { analysis } \\
\text { P-value }\end{array}$} \\
\hline & & & & \\
\hline Gender & & & 0.296 & \\
\hline Male & $430(76.0)$ & $74(7 \mid .2)$ & & \\
\hline Female & $136(24.0)$ & $30(28.8)$ & & \\
\hline Age, years & & & $<0.001^{*}$ & $<0.001^{*}$ \\
\hline$<65$ & $30 \mathrm{I}(53.2)$ & $14(13.5)$ & & \\
\hline$\geq 65$ & $265(46.8)$ & $90(86.5)$ & & \\
\hline $\mathrm{BMI}, \mathrm{kg} / \mathrm{m}^{2}$ & & & $<0.001^{*}$ & $0.03^{*}$ \\
\hline$<18$ & $24(4.2)$ & $16(15.5)$ & & \\
\hline$\geq 18$ & $54 I(95.8)$ & $87(84.5)$ & & \\
\hline ASA & & & $0.001^{*}$ & 0.744 \\
\hline $\mathrm{I}$ & $80(14.1)$ & $6(5.8)$ & & \\
\hline 2 & $400(70.7)$ & $67(54.8)$ & & \\
\hline 3 & $85(15.0)$ & $30(28.8)$ & & \\
\hline 4 & $I(0.2)$ & $I(I .0)$ & & \\
\hline NRS & & & $<0.001^{*}$ & $0.001^{*}$ \\
\hline 1 & $208(36.8)$ & $4(3.8)$ & & \\
\hline 2 & $162(28.6)$ & $24(23.1)$ & & \\
\hline 3 & $111(19.6)$ & $31(29.8)$ & & \\
\hline$>3$ & $85(15.0)$ & $45(43.3)$ & & \\
\hline Diabetes & & & $0.006^{*}$ & $0.008^{*}$ \\
\hline No & $510(90.1)$ & $84(80.8)$ & & \\
\hline Yes & $56(9.9)$ & $20(19.2)$ & & \\
\hline Surgery history & & & $0.036^{*}$ & 0.577 \\
\hline No & $459(81.1)$ & $75(72.1)$ & & \\
\hline Yes & $107(18.9)$ & $29(27.9)$ & & \\
\hline Histologic type & & & $0.042^{*}$ & 0.653 \\
\hline Differentiated & $200(35.4)$ & $26(25.0)$ & & \\
\hline Undifferentiated & $365(64.6)$ & $78(75.0)$ & & \\
\hline Albumin, $g / L$ & & & $<0.001^{*}$ & 0.006 \\
\hline$\geq 35$ & $452(79.9)$ & $46(44.2)$ & & \\
\hline$<35$ & $114(20.1)$ & $58(55.8)$ & & \\
\hline Hemoglobin, g/L & & & $<0.001^{*}$ & 0.628 \\
\hline$\geq 100$ & $47 \mid(83.2)$ & $62(59.6)$ & & \\
\hline$<100$ & $95(16.8)$ & $42(40.4)$ & & \\
\hline NLR & & & $<0.001^{*}$ & $0.006^{*}$ \\
\hline Low & $395(69.8)$ & $39(37.5)$ & & \\
\hline High & $|7|(30.2)$ & $65(62.5)$ & & \\
\hline PLR & & & $<0.001^{*}$ & $0.046^{*}$ \\
\hline Low & $179(31.6)$ & $\mathrm{II}(10.6)$ & & \\
\hline High & $387(68.4)$ & $93(89.4)$ & & \\
\hline LPLR & & & $<0.001^{*}$ & 0.137 \\
\hline Low & $450(80.5)$ & $56(54.4)$ & & \\
\hline High & $109(19.5)$ & $47(45.6)$ & & \\
\hline Node status & & & $0.017^{*}$ & 0.842 \\
\hline No & $270(48.1)$ & $32(31.7)$ & & \\
\hline NI & $96(17.1)$ & $21(20.8)$ & & \\
\hline N2 & $104(18.5)$ & $23(22.8)$ & & \\
\hline N3 & $91(16.2)$ & $25(24.8)$ & & \\
\hline T stage & & & $<0.001^{*}$ & 0.733 \\
\hline TI & $163(29.1)$ & 10(9.9) & & \\
\hline $\mathrm{T} 2$ & $67(12.0)$ & $12(11.9)$ & & \\
\hline $\mathrm{T} 3$ & $116(20.7)$ & $26(25.7)$ & & \\
\hline $\mathrm{T} 4$ & $214(38.2)$ & $53(52.5)$ & & \\
\hline
\end{tabular}

Notes: *Statistically significant $(P<0.05)$.

Abbreviations: ASA, American Society of Anesthesiologists; BMI, body mass index; LPLR, large platelet/lymphocyte ratio; NLR, neutrophil/lymphocyte ratio; NRS, nutritional risk screening; PLR, platelet/lymphocyte ratio. 
Table 4 Clinical characteristics of validation cohort (\%)

\begin{tabular}{|c|c|c|c|c|}
\hline Factors & $\begin{array}{l}\text { Total, } \\
162(\%)\end{array}$ & $\begin{array}{l}\text { Low PLR, } \\
47(\%)\end{array}$ & $\begin{array}{l}\text { High PLR, } \\
\text { I I } 5 \text { (\%) }\end{array}$ & $P$-value \\
\hline Age, years & & & & 0.493 \\
\hline$<65$ & $83(51.2)$ & $22(46.8)$ & $61(53.0)$ & \\
\hline$\geq 65$ & $79(48.8)$ & $25(53.2)$ & $54(47.0)$ & \\
\hline Gender & & & & 0.708 \\
\hline Male & $112(69.1)$ & $34(72.3)$ & $78(67.8)$ & \\
\hline Female & $50(30.9)$ & $13(27.7)$ & $37(32.2)$ & \\
\hline BMI, $\mathrm{kg} / \mathrm{m}^{2 \#}$ & & & & 0.449 \\
\hline$<18$ & $9(5.6)$ & $I(2 . I)$ & $8(7.0)$ & \\
\hline$\geq 18$ & $153(94.4)$ & $46(97.9)$ & $107(93.0)$ & \\
\hline ASA & & & & 0.662 \\
\hline 1 & $47(29.0)$ & $15(3 \mid .9)$ & $32(27.8)$ & \\
\hline 2 & $100(61.7)$ & $29(61.7)$ & $71(6 \mid .7)$ & \\
\hline 3 & $15(9.3)$ & $3(6.4)$ & $12(10.5)$ & \\
\hline NRS & & & & 0.706 \\
\hline 1 & 63(38.9) & $18(38.3)$ & $45(39.1)$ & \\
\hline 2 & $47(29.0)$ & $16(34.0)$ & $3 I(27.0)$ & \\
\hline 3 & $24(14.8)$ & $7(14.9)$ & $17(14.8)$ & \\
\hline$>3$ & $28(17.3)$ & $6(12.8)$ & $22(19.1)$ & \\
\hline Diabetes & & & & $0.044^{*}$ \\
\hline Yes & $21(13.0)$ & $10(21.3)$ & $\mathrm{II}(9.6)$ & \\
\hline No & $|4|(87.0)$ & $37(78.7)$ & $104(90.4)$ & \\
\hline Hypertension & & & & 0.462 \\
\hline No & $109(67.3)$ & $34(72.3)$ & $75(65.2)$ & \\
\hline Yes & $53(32.7)$ & $13(27.7)$ & $40(34.8)$ & \\
\hline Albumin, $g / L$ & & & & 0.314 \\
\hline$<35$ & $27(16.7)$ & $10(21.3)$ & $17(14.8)$ & \\
\hline$\geq 35$ & $135(83.3)$ & $37(78.7)$ & $98(85.2)$ & \\
\hline Hemoglobin, g/L & & & & $<0.00$ I* \\
\hline$<100$ & $36(22.2)$ & $22(46.8)$ & $14(12.2)$ & \\
\hline$\geq 100$ & $126(77.8)$ & $25(53.2)$ & $101(87.8)$ & \\
\hline Histologic type & & & & 0.057 \\
\hline Differentiated & $129(79.6)$ & $33(70.2)$ & $96(83.5)$ & \\
\hline Undifferentiated & $33(20.4)$ & $14(29.8)$ & $19(16.5)$ & \\
\hline Node status & & & & $0.35 I$ \\
\hline No & $79(49.7)$ & $20(43.5)$ & $59(52.2)$ & \\
\hline NI & $28(17.6)$ & $\mathrm{II}(23.9)$ & $17(15.1)$ & \\
\hline N2 & $19(11.9)$ & $8(17.4)$ & $\mathrm{II}(9.7)$ & \\
\hline N3 & $33(20.8)$ & $7(15.2)$ & $26(23.0)$ & \\
\hline T stage & & & & 0.924 \\
\hline $\mathrm{Tis} / \mathrm{TI}$ & $44(27.2)$ & $13(27.7)$ & $3 I(26.9)$ & \\
\hline $\mathrm{T} 2$ & $27(16.7)$ & $9(19.1)$ & $18(15.7)$ & \\
\hline T3 & $28(I 7.3)$ & $7(14.9)$ & $21(18.3)$ & \\
\hline $\mathrm{T} 4$ & $63(38.8)$ & $18(38.3)$ & $45(39.1)$ & \\
\hline Sarcopenia & & & & $0.035^{*}$ \\
\hline No & $135(83.3)$ & $44(93.6)$ & $91(79.1)$ & \\
\hline Yes & $27(16.7)$ & $3(6.4)$ & $24(20.9)$ & \\
\hline Muscle mass, $\mathrm{cm}^{2} / \mathrm{m}^{2}$ (IQR) & $\begin{array}{l}45.64 \\
(39.58-50.53)\end{array}$ & $\begin{array}{l}46.17 \\
(42.36-50.38)\end{array}$ & $\begin{array}{l}45.42 \\
(38.99-50.73)\end{array}$ & \\
\hline $\begin{array}{l}\text { Grip strength, kg } \\
\text { (IQR) }\end{array}$ & $\begin{array}{l}27.33 \\
(21.98-33.30)\end{array}$ & $\begin{array}{l}26.90 \\
(22.40-33.30)\end{array}$ & $\begin{array}{l}27.51 \\
(21.90-33.30)\end{array}$ & \\
\hline $\begin{array}{l}\text { Gait speed, } \mathrm{m} / \mathrm{s} \\
\text { (IQR) }\end{array}$ & $\begin{array}{l}1.08 \\
(0.87-1.28)\end{array}$ & $\begin{array}{l}1.04 \\
(0.86-1.22)\end{array}$ & $\begin{array}{l}1.09 \\
(0.88-1.30)\end{array}$ & \\
\hline
\end{tabular}

Notes: The values given are number of patients unless indicated otherwise. *Statistically significant $(P<0.05)$; \#the data are partially lost for various reasons.

Abbreviations: ASA, American Society of Anesthesiologists; BMI, body mass index; IQR, interquartile range; NLR, neutrophil/lymphocyte ratio; NRS, nutritional risk screening; PLR, platelet/lymphocyte ratio. 
the hypothesis that host-related SIR increases the incidence of sarcopenia. Furthermore, there were direct relationships between age, NRS, albumin, BMI, and diabetes with sarcopenia. Inflammation may cause malnutrition status; nevertheless, we suggested that inflammation and malnutrition jointly lead to sarcopenia in this study. ${ }^{31}$ Sarcopenia may be a good indicator of malnutrition. Malnutrition, particularly protein deficiency, has been reported to cause immune impairment and reduce body strength. Conversely, although a pilot study had shown that the accuracy of LPLR was superior to that of PLR in patients with cancer, ${ }^{22}$ justifying the inclusion of LPLR as one of our preoperative factors, this measure was found to be irrelevant to sarcopenia $(P=0.137)$.

Age-related factors, inactivity, inappetence, and insufficient nutritional intake are defined as mechanisms that alter skeletal muscle strength. ${ }^{32}$ Moreover, several lines of evidence show that the host-related inflammatory response reflects the body composition. Another report has suggested that the inflammatory response, previously acknowledged as a marker of prognosis in gastrointestinal cancer patients, is related with the basic characteristics of muscle wastage, for instance, decreased quality of life or increased risk of morbidity and mortality. ${ }^{33}$ Our study shows that a similar relationship exists in gastric cancer: preoperative elevated NLR and PLR were associated with sarcopenia. In this study, elevated NLR and PLR were observed in half of the sarcopenia patients $(57.7 \%)$. The finding that inflammation is associated with sarcopenia is consistent with a well-established prior literature on non-metastatic colon carcinoma. ${ }^{21}$

The close correlation between sarcopenia and short-term and long-term prognosis of gastric cancer highlights the need for early nutritional status assessment. ${ }^{11,12}$ Given the complex diagnosis of sarcopenia, the presence of motor dysfunction or the lack of clinically acquired computed tomography images will misjudge the nutrition status of gastric cancer patients. Using PLR as primary screening test (a sensitivity of $91.3 \%$ ) aids with the early, simple, and convenient identification of sarcopenia, which may facilitate the use of therapeutic intervention to ensure a successful perioperative management and postoperative rehabilitation, a high quality of life, and a longer likelihood of survival. Such treatments might include taking anti-inflammatory drugs, ${ }^{34}$ following a Mediterranean-style diet, ${ }^{35}$ and/or partaking in regular resistance exercise, which are evidenced to be valid and reliable in keeping and enhancing muscle mass and function in cancer patients, improve life quality, and effectively prolong survival. ${ }^{36-38}$
This study was strengthened by its large sample size and prospective data collection. However, our study has several limitations. Firstly, we were unable to disentangle the network of two-way relationships among inflammation, sarcopenia, and cancer. Further research is needed to address this issue. Secondly, this was a single institution study. A multicenter prospective study including other regions is essential to overcome this limitation and broaden the generalizability of the study results. Thirdly, we defined 18 as the cutoff point of BMI, by which a group of people with sarcopenic obesity may have been ignored. However, in Asia, the incidence of sarcopenic obesity is low, with only rare individuals being ascertained in this study.

\section{Conclusion}

The present study showed that PLR and NLR could be related with sarcopenia in gastric cancer patients. The understanding of their mechanisms should be helpful as potential therapeutic targets. Furthermore, PLR, NLR, and other indicators may aid in distinguishing gastric cancer patients with sarcopenia, which would likely be helpful for further planning of timely nutritional intervention prior to surgery. Together with our findings, additional studies on NLR and PLR measures in combination with other known indicators should be explored to predict the risk of malnutrition in patients with gastric cancer in the future.

\section{Acknowledgments}

The authors thank all the participants in this study and the members of our research team. This work was supported by the Science and Technology Incubation Program of Wenzhou Medical University, Zhejiang Province, China (FHY2014039) and Project of Wenzhou Municipal Science and Technology Bureau, Zhejiang Province, China (Y20170104).

\section{Ethics approval and consent to participate}

All participants provided their written informed consent, and the protocol for this study was approved by the ethics committee of The First Affiliated Hospital of Wenzhou Medical University (2014 NO.063).

\section{Author contributions}

All authors contributed to data analysis, drafting and revising the article, gave final approval of the version to be published, and agree to be accountable for all aspects of the work. 


\section{Disclosure}

The authors report no conflicts of interest in this work.

\section{References}

1. Torre LA, Bray F, Siegel RL, Ferlay J, Lortet-Tieulent J, Jemal A. Global cancer statistics, 2012. CA Cancer J Clin. 2015;65(2):87-108.

2. Kim Y, Ejaz A, Spolverato G, et al. Conditional survival after surgical resection of gastric cancer: a multi-institutional analysis of the us gastric cancer collaborative. Ann Surg Oncol. 2015;22(2):557-564.

3. Ferlay J, Soerjomataram I, Dikshit R, et al. Cancer incidence and mortality worldwide: sources, methods and major patterns in GLOBOCAN 2012. Int J Cancer. 2015;136(5):E359-E386.

4. Esfahani A, Somi MH, Asghari Jafarabadi M, et al. A new score for screening of malnutrition in patients with inoperable gastric adenocarcinoma. Jpn J Clin Oncol. 2017;47(6):475-479.

5. Morley JE, Baumgartner RN, Roubenoff R, Mayer J, Nair KS. Sarcopenia. J Lab Clin Med. 2001;137(4):231-243.

6. Fuggle N, Shaw S, Dennison E, Cooper C. Sarcopenia. Best Pract Res Clin Rheumatol. 2017;31(2):218-242.

7. Takama T, Okano K, Kondo A, et al. Predictors of postoperative complications in elderly and oldest old patients with gastric cancer. Gastric Cancer. 2015;18(3):653-661.

8. Tsujinaka T, Sasako M, Yamamoto S, et al. Influence of overweight on surgical complications for gastric cancer: results from a randomized control trial comparing D2 and extended para-aortic D3 lymphadenectomy (JCOG9501. Ann Surg Oncol. 2007;14(2):355-361.

9. Park DJ, Lee HJ, Kim HH, Yang HK, Lee KU, Choe KJ. Predictors of operative morbidity and mortality in gastric cancer surgery. Br J Surg. 2005;92(9):1099-1102.

10. Kim MC, Kim W, Kim HH, et al. Risk factors associated with complication following laparoscopy-assisted gastrectomy for gastric cancer: a largescale Korean multicenter study. Ann Surg Oncol. 2008;15(10):2692-2700.

11. Wang SL, Zhuang CL, Huang DD, et al. Sarcopenia Adversely Impacts Postoperative Clinical Outcomes Following Gastrectomy in Patients with Gastric Cancer: A Prospective Study. Ann Surg Oncol. 2016;23(2):556-564.

12. Zhuang CL, Huang DD, Pang WY, et al. Sarcopenia is an Independent Predictor of Severe Postoperative Complications and Long-Term Survival After Radical Gastrectomy for Gastric Cancer: Analysis from a Large-Scale Cohort. Medicine (Baltimore). 2016;95(13):e3164.

13. Grivennikov SI, Greten FR, Karin M. Immunity, inflammation, and cancer. Cell. 2010;140(6):883-899.

14. Balkwill F, Mantovani A. Inflammation and cancer: back to Virchow? Lancet. 2001;357(9255):539-545.

15. Coussens LM, Werb Z. Inflammation and cancer. Nature. 2002; 420(6917):860-867.

16. Templeton AJ, Ace O, Mcnamara MG, et al. Prognostic role of platelet to lymphocyte ratio in solid tumors: a systematic review and meta-analysis. Cancer Epidemiol Biomarkers Prev. 2014;23(7):1204-1212.

17. Templeton AJ, Mcnamara MG, Šeruga B, et al. Prognostic role of neutrophil-to-lymphocyte ratio in solid tumors: a systematic review and meta-analysis. J Natl Cancer Inst. 2014;106(6):dju124.

18. Zhou X, Du Y, Huang Z, et al. Prognostic value of PLR in various cancers: a meta-analysis. PLoS One. 2014;9(6):e101119.

19. Guthrie GJ, Charles KA, Roxburgh CS, Horgan PG, Mcmillan DC, Clarke SJ. The systemic inflammation-based neutrophil-lymphocyte ratio: experience in patients with cancer. Crit Rev Oncol Hematol. 2013;88(1):218-230.

20. Pang W, Lou N, Jin C, et al. Combination of preoperative platelet/ lymphocyte and neutrophil/lymphocyte rates and tumor-related factors to predict lymph node metastasis in patients with gastric cancer. Eur J Gastroenterol Hepatol. 2016;28(5):493-502.
21. Feliciano EMC, Kroenke CH, Meyerhardt JA, et al. Association of Systemic Inflammation and Sarcopenia With Survival in Nonmetastatic Colorectal Cancer: Results From the C SCANS Study. JAMA Oncol. 2017;3(12):e172319.

22. Seretis C, Seretis F, Lagoudianakis E, Politou M, Gemenetzis G, Salemis NS. Enhancing the accuracy of platelet to lymphocyte ratio after adjustment for large platelet count: a pilot study in breast cancer patients. Int J Surg Oncol. 2012;2012:7.

23. Cruz-Jentoft AJ, Baeyens JP, Bauer JM, et al. Sarcopenia: European consensus on definition and diagnosis: Report of the European Working Group on Sarcopenia in Older People. Age Ageing. 2010;39(4): 412-423.

24. Kondrup J, Rasmussen HH, Hamberg O, Stanga Z, Ad Hoc ESPEN Working Group, Ad Hoc ESPEN Working Group. Nutritional risk screening (NRS 2002): a new method based on an analysis of controlled clinical trials. Clin Nutr. 2003;22(3):321-336.

25. Sano T, Aiko T. New Japanese classifications and treatment guidelines for gastric cancer: revision concepts and major revised points. Gastric Cancer. 2011;14(2):97-100.

26. Chen LK, Liu LK, Woo J, et al. Sarcopenia in Asia: consensus report of the Asian Working Group for Sarcopenia. J Am Med Dir Assoc. 2014;15(2):95-101.

27. Mourtzakis M, Prado CM, Lieffers JR, Reiman T, Mccargar LJ, Baracos VE. A practical and precise approach to quantification of body composition in cancer patients using computed tomography images acquired during routine care. Appl Physiol Nutr Metab. 2008;33(5):997-1006.

28. Lieffers JR, Bathe OF, Fassbender K, Winget M, Baracos VE. Sarcopenia is associated with postoperative infection and delayed recovery from colorectal cancer resection surgery. $\mathrm{Br} J$ Cancer. 2012;107(6):931-936.

29. Prado CM, Lieffers JR, Mccargar LJ, et al. Prevalence and clinical implications of sarcopenic obesity in patients with solid tumours of the respiratory and gastrointestinal tracts: a population-based study. Lancet Oncol. 2008;9(7):629-635.

30. Fluss R, Faraggi D, Reiser B. Estimation of the Youden Index and its associated cutoff point. Biom J. 2005;47(4):458-472.

31. Gonda K, Shibata M, Sato Y, et al. Elevated neutrophil-to-lymphocyte ratio is associated with nutritional impairment, immune suppression, resistance to S-1 plus cisplatin, and poor prognosis in patients with stage IV gastric cancer. Mol Clin Oncol. 2017;7(6):1073-1078.

32. Fearon K, Arends J, Baracos V. Understanding the mechanisms and treatment options in cancer cachexia. Nat Rev Clin Oncol. 2013;10(2):90-99.

33. Richards CH, Roxburgh CS, Macmillan MT, et al. The relationships between body composition and the systemic inflammatory response in patients with primary operable colorectal cancer. PLoS One. 2012;7(8):e41883.

34. Ewaschuk JB, Almasud A, Mazurak VC. Role of n-3 fatty acids in muscle loss and myosteatosis. Appl Physiol Nutr Metab. 2014;39(6):654-662.

35. Yarla NS, Polito A, Peluso I. Effects of Olive Oil on TNF- $\alpha$ and IL-6 in Humans: Implication in Obesity and Frailty. Endocr Metab Immune Disord Drug Targets. 2018;18(1):63-74.

36. Focht BC, Clinton SK, Devor ST, et al. Resistance exercise interventions during and following cancer treatment: a systematic review. J Support Oncol. 2013;11(2):45-60.

37. Hardee JP, Porter RR, Sui X, et al. The effect of resistance exercise on all-cause mortality in cancer survivors. Mayo Clin Proc. 2014;89(8):1108-1115.

38. Strasser B, Steindorf K, Wiskemann J, Ulrich CM. Impact of resistance training in cancer survivors: a meta-analysis. Med Sci Sports Exerc. 2013;45(11):2080-2090. 


\section{Publish your work in this journal}

Cancer Management and Research is an international, peer-reviewed open access journal focusing on cancer research and the optimal use of preventative and integrated treatment interventions to achieve improved outcomes, enhanced survival and quality of life for the cancer patient. The manuscript management system is completely online and includes a very quick and fair peer-review system, which is all easy to use. Visit http://www.dovepress.com/testimonials.php to read real quotes from published authors.

Submit your manuscript here: https://www.dovepress.com/cancer-management-and-research-journal 